\title{
LA INTEGRACIÓN SUDAMERICANA EN LA ENCRUCIJADA ENTRE LA IDEOLOGÍA Y EL PRAGMATISMO
}

\author{
The South American integration at the crossroads \\ between ideology and pragmatism
}

Míriam Gomes Saraiva

Programa de Posgrado en Relaciones Internacionales

Universidad del Estado de Rio de Janeiro

miriamgsaraiva@gmail.com

https://orcid.org/0000-0001-8212-5509

Lorena Granja Hernández

Programa de Posgrado en Relaciones Internacionales

Universidad del Estado de Rio de Janeiro

lorenagranja@gmail.com

https://orcid.org/0000-0002-4314-0818

Recibido: $16 / 10 / 2018$

Aceptado: 21/02/2019

\begin{abstract}
Resumen: El trabajo plantea el abordaje conceptual de la gobernanza regional para reflexionar sobre la encrucijada en que se encuentra la integración sudamericana. Ese concepto es articulado, en un continuo en el que los diferentes actores interactúan-cooperan-coordinan-integran, con dos dimensiones transversales: la regionalidad y la ideología. La primera parte del texto considera los términos utilizados en los debates conceptuales sobre regionalismo, integración, regionalización, regionalidad y gobernanza. La segunda, se adentra en el análisis de los patrones de convergencia o divergencia ideológica que se han sucedido entre los gobiernos de la región; plantea que las prácticas y procesos instaurados para la gobernanza regional se articulan entre la ideología y el pragmatismo. En las reflexiones finales se adelanta la futura agenda de investigación.
\end{abstract}

Palabras clave: regionalismo, América Latina, gobernanza regional, procesos de integración regional, política exterior. 


\begin{abstract}
The paper proposes a conceptual approach based on regional governance to think about the crossroads in which the South American integration process is now. This concept is articulated in a continuum in which the different actors interact-cooperate-coordinate-integrate and has also two transversal dimensions: the regionness and the ideology. The first section analyzes the conceptual debates about regionalism, integration, regionalization, regionness and governance. The second one, identifies the patterns of ideological convergence or divergence that have been taken place between the governments at the region; the claim is that the practices and processes established to the regional governance are articulated between ideology and pragmatism. In the final reflections, the future research agenda is advanced.
\end{abstract}

Keywords: regionalism, Latin America, regional governance, process of regional integration. foreign policy. 


\section{Introducción}

La integración regional en América del Sur ha sido tratada, pensada y construida por académicos, políticos y actores socioeconómicos de maneras disímiles a lo largo de su trayectoria. Las diferencias en la forma en cómo es concebida ontológicamente provienen necesariamente de distintas posturas ideológicas sobre el carácter que debería asumir por ese trípode de actores implicados que, frente al contexto y según el momento, se articulan con límites pragmáticos. En tal sentido, la integración ha sido entendida o bien como un fin en sí mismo; una especie de utopía fundacional donde se depositan esperanzas de cambio, un instrumento para alcanzar objetivos ideológicamente concomitantes, fueran estos de unificación, cooperación regional, inserción internacional conjunta, crecimiento económico, desarrollo o cohesión social. Siempre ha habido diferentes posibilidades y combinaciones; actualmente, frente a los diversos cambios políticos e ideológicos que se manifiestan en la región, la integración regional y sus formatos se encuentra delante de una encrucijada.

El presente artículo propone reflexionar conceptualmente sobre esas diferentes maneras de concebir la articulación de la acción colectiva a nivel regional, desde las prácticas a las ideas; propone un esquema analítico que define a la gobernanza regional como un concepto capaz de captar las diferencias de comportamientos de corte ideológico y pragmático en la concepción y articulación del regionalismo sudamericano.

Las experiencias integracionistas construidas a lo largo de los años, y sus principales consecuencias, han sido relatadas por esos tres sujetos (académicos, políticos y actores socioeconómicos) con dejos de decepción, cuando no de completa incredulidad, tanto sea en el medio académico, político o en los de comunicación - e independientemente de si son a favor o en contra, siempre ha habido críticas por insuficiencias. Sin embargo, entre la ideología y el pragmatismo de sus actores, la integración regional en la práctica es construida cotidiana y paulatinamente, con altos y bajos, idas y venidas. El pasaje de siglo dejó nuevas experiencias en Sudamérica, muchas de ellas enmarcadas en grandes retóricas político-ideológicas (ALBA), otras resignificadas en sus aspectos funcionales (Mercosur), o con elementos de innovación institucional y política (Unasur). Se considera que los esfuerzos de construcción discursiva, identitaria e institucional que se han sucedido de manera un tanto alternada, no lineal y superpuestos en la trayectoria histórica de los regionalismos sudamericanos, son la base para la construcción de capacidades que hagan de la integración regional un camino sinuoso, pero sin vuelta atrás y con destino seguro. 
Sea como fin o como instrumento, se propone pensar conceptualmente sobre los esfuerzos realizados para la construcción de proyectos de integración regional; identificar cuáles son los saldos positivos de esas experiencias con especial foco en sus consecuencias o legados para la construcción de demandas regionales que les den autosostenibilidad a tales iniciativas. Es decir, que mantengan a las experiencias integracionistas funcionando y construyendo regionalidad conjuntamente a lo largo del tiempo. Aunque no se hace un análisis exhaustivo de los diferentes marcos institucionales, se propone considerar particularmente su importancia para la construcción de capacidades regionales, demandas por gobernanza regional multinivel que tienen como protagonistas a diversos actores (estatales, sociales, públicos y privados). En ese sentido, y como se parte del análisis institucional para poner el foco en los actores, se plantea que ideológicamente que estos son muy diversos; aunque pragmáticamente, tengan más puntos de convergencia que los esperados.

La hipótesis es que aun cuando ideológicamente haya diferentes maneras de entender a la integración regional, las experiencias pragmáticas dejan de manifiesto que estas pueden, eventualmente, no converger, paralizarse ante momentos de crisis o incertidumbres; pero que no sucumben en la conformación identitaria, ni política regional. Por el contrario, aun cuando el discurso retórico sea el de la des(integración) podrían permear prácticas de gobernanza regional que mantengan cierto nivel de coordinación pragmática entre los actores. En períodos de alta convergencia ideológica, se alcanzan mayores resultados en términos integracionistas; sea cual sea su orientación política. El pragmatismo también ha reinado en dichos períodos; y buena parte de sus resultados pueden ser incluso las bases del siguiente esfuerzo regional. En períodos donde la convergencia ideológica ha decaído; los escenarios pragmáticos se multiplican. Aparecen cambios más o menos bruscos o aparentes parálisis en las decisiones; en este sentido se plantea que pueden generarse procesos que autorreproduzcan regionalización: patrones de reproducción de prácticas en niveles múltiples que demanden la continuidad, construcción o reproducción de (algún grado de) gobernanza regional.

Una de las bases de la reflexión presentada es el análisis de la política exterior - sobre todo los análisis comparados - que hn abordado ya en gran medida las diferencias entre la retórica del discurso y la práctica de las políticas. En ese sentido, Gardini (2011) hizo una útil diferenciación entre politica exterior pragmática e ideológica. La política exterior pragmática tendría su mérito en la utilidad, practicidad y ejecutabilidad; en vez de estar orientada doctrinariamente por principios prefijados. Está asociada a un horizonte político de mediano plazo y no necesariamente a un determinado gobierno. Por su parte, la política externa ideológica enfatiza soluciones de principios, normativos o doctrinales, no necesariamente 
vinculados al mérito de la política e inclusive poco prácticos. En la visión del autor, elementos característicos de los dos tipos de política exterior son articulados con frecuencia por los países de la región. Su relativo suceso no es necesariamente consecuencia de un perfil ideológico o pragmático en la formulación; ambas definiciones se aplican a las motivaciones de la política no a los resultados.

En este marco de reflexiones, es importante efectuar una discusión conceptual sobre las diferentes maneras de entender la integración regional y su consecuente construcción. Se propone pensar, en primer lugar, en los abordajes teóricos que han pautado su estudio. Además, se analizan las distintas ideas de integración que han predominado en la región y se establecen algunos puntos conceptuales básicos (comunes) para futuras reflexiones. Fundamentalmente, se entiende a la gobernanza regional como el principal saldo de los diferentes marcos ideológicos en los que la integración ha sido planteada; y se utiliza dicho concepto como marco de referencia analítico. En ese transcurso, no se pretende exhaustividad, aunque sí tejer el hilo argumental para pensar el modelo/esquema conceptual que se entiende como el marco analítico plausible de ser replicado o adoptado para otras regiones, proyectos y contextos.

Posteriormente, las hipótesis mencionadas encima son consideradas en su contexto empírico bajo una de las dimensiones del marco conceptual propuesto: la convergencia/divergencia ideológica y sus opciones pragmáticas. Tal dimensión se identifica en la relación entre los gobiernos de la región de manera continua, aunque no constante; con períodos de mayor o menor convergencia ideológica en los que se tejieron soluciones más o menos pragmáticas para construir gobernanza regional. Se busca identificar tales patrones de convergencia/divergencia planteados en la trayectoria contemporánea sudamericana, sin pretensión de exhaustividad. Esa contextualización permite examinar con cierta perspectiva la plausibilidad de las hipótesis planteadas; parte del argumento se sustenta en la construcción institucional de prácticas que están limitadas por la ideología —integración - y sus formas de moldearla - pragmatismo- que se identifican a lo largo de la trayectoria del regionalismo sudamericano. Es interesante, también, ver cómo es su evolución, su variación y si hay patrones establecidos entre y ante escenarios ideológica y contextualmente distintos.

Por fin, en las reflexiones finales se adelanta la futura agenda de investigación que, a partir de la aplicación del esquema conceptual planteado en el artículo podrá dar continuidad al debate. El artículo contribuye en varios sentidos con la propuesta del dosier; se propone una formulación conceptual más amplia de la integración regional, a partir de una reconceptualización de la gobernanza regional como concepto clave para tal análisis. Además, concomitante al proceso de formulación del marco analítico, algunas reflexiones empíricas del contexto 
regional sudamericano son introducidas tangencialmente: el análisis comparado de las políticas exteriores y sus consecuencias; los micro procesos de la toma de decisiones y la construcción del regionalismo.

\section{Concepciones de integración regional en la América del Sur contemporánea}

La trayectoria y experiencia sudamericana abren oportunidades para analizar la integración de manera original; muchos han sido los académicos que proponen abordajes ricos conceptual y empíricamente. Por tales motivos, no caben análisis exclusivamente centrados en conceptos desarrollados a partir de la experiencia europea de integración, así como tampoco serían relevantes ciertas comparaciones. De cualquier manera, existen algunas conceptualizaciones sobre el proceso de integración europeo que merecen ser evaluados en sus ventajas analíticas; la acumulación conceptual es una de las más importantes tareas de las investigaciones.

En esta sección se analizan abordajes teóricos planteados por expertos en torno al debate sobre integración. Se recorren algunos de los argumentos propuestos para construir el hilo argumental. En ese sentido, la construcción no es neutral. Primero, se sugiere esclarecer algunos de los hiperreferidos conceptos del debate: regionalismo, integración regional y regionalización. Luego, se presenta una propuesta de conceptualización de la gobernanza regional como término que permitiría superar la confusión conceptual a partir de su entendimiento como instrumento analítico.

\subsection{Regionalismo, integración regional, regionalización y la idea de regionalidad}

Al no existir definiciones claras o consensuadas sobre el significado de estos términos han sido tratados de forma diferente por académicos y políticos, lo que constituye un ejemplo de conducta pragmática y genera confusiones.

Identificado necesariamente con la idea de región, que implica directamente espacios geográficamente referenciados, compuesto por diferentes formas de interacción que toman lugar dentro de sus límites o representándola fuera de ella (dimensión relacional), muchos académicos adoptaron el término regionalismo para definir diferentes acepciones empíricas de la integración. Ambos términos han sido considerados como sinónimos por algunos analistas; en otros casos, han sido bien diferenciados teóricamente; también, se han confundido las diferentes terminologías empleadas, con los rumbos que los distintos proyectos han tomado, dimensión ideológico-pragmática. De hecho, tanto su 
necesaria vinculación empírica, como su intrínseca relación con las dimensiones geo-referencial y relacional, han motivado tal confusión conceptual.

Pía Riggirozzi (2010) consideraba como regionalismo a las tendencias encontradas en determinados momentos hacia uno u otro modelo de integración; mientras que integración regional denotaría características propias de cada proyecto específico ensayado. En acuerdo con tal posición, se adopta la idea de Fawcett $(2013$, p. 8) que enuncia que el regionalismo debe ser entendido bajo la lupa del método histórico comparativo; puesto que sería a partir de su evolución práctica que tomaría diferentes formas y significados; dicha evolución no lineal ni uniforme es fruto de los factores internos y externos que le dan sustento. Asimismo, el regionalismo debe verse en sus procesos históricos; sin perder de vista el significado y la construcción contextual: a pesar de las crisis y estancamientos institucionales, el regionalismo ha estado siempre presente en el discurso e imaginario latinoamericano (Briceño-Ruiz y Rivarola, 2013).

Coexisten diferentes ideas de integración en la región que se han venido articulando y alternando de forma poco armoniosa (Briceño-Ruiz, 2017b), muchas veces pujando, incluso, en sentidos ideológicamente opuestos (Gómez-Mera, 2015). Estas ideas han sido identificadas con el término regionalismo; y cada uno de sus diferentes precursores (entre los que se encuentran analistas, actores políticos e institucionales) han adjetivado al regionalismo de distinta forma, lo que daría lugar a llamarlos regionalismos sudamericanos, en plural. Tal vez esas ideas integracionistas no sean tan polarizadas como es costumbre pensarlas desde los diferentes proyectos regionales que se han sucedido a lo largo del tiempo; ni sean, inclusive, tan diferentes en sus resultados. Se coincide con Dabène (2009) al afirmar que hay consistencias y procesos de resiliencia en esa trayectoria de coexistencia de altos y bajos en los regionalismos; asunto retomado por Briceño-Ruiz y Rivarola (2013) al discutir las raíces históricas e ideológicas profundas del regionalismo latinoamericano, frecuentemente olvidadas en los análisis excesivamente centrados en el presente.

Nolte y Comini (2016) consideran al regionalismo como resultado de las preferencias construidas conjuntamente durante las negociaciones, reflejo de los consensos alcanzados. Por tanto, no necesariamente supone un fracaso la no consecución de los objetivos propuestos. En oposición a los análisis que sustentan una supuesta ineficacia o ineficiencia en los proyectos de integración sudamericanos, al no ser consecuentes con metas inicial o externamente propuestas; esta perspectiva prioriza al pragmatismo y da sustento a la idea de que la convergencia ideológica es una condición suficiente para la construcción de gobernanza regional; aunque no necesaria. En diálogo con el abordaje que apunta como problema para 
el regionalismo la variedad de instituciones y, consecuentemente, de lealtades (Malamud y Gardini, 2012), se coincide con la perspectiva de que la sobreposición de organizaciones puede ser vista como un ejemplo de altos patrones de convergencia exitosa por parte de sus actores políticos que, sumado a las prácticas adoptadas - entre las que se identifican la flexibilización institucional y la segmentación organizacional (Gómez-Mera, 2015; Nolte y Comini, 2016, p. 547)-, se transforman en una característica pragmática del regionalismo sudamericano.

Por otra parte, Hurrell (1995) proponía dotar de contenido ontológico al regionalismo y lo descomponía en cinco categorías que, aunque pertenecientes a distintas dimensiones analíticas, apuntan las principales características empíricas del fenómeno. Entre ellas, merecen destaque el proceso de regionalización espontánea y la construcción paulatina de cohesión regional, cuando la región ejerce un papel definidor de las relaciones entre los Estados; o cuando la región conforma la base sobre la que se organizan políticas específicas. Tal concepción, a pesar de su linealidad al suponer una consecución de etapas entre las categorías, es importante puesto que identifica diferentes dimensiones analíticas que se pretenden complementar en dos sentidos con el abordaje de la gobernanza aquí propuesto: al ver a la convergencia ideológica como una dimensión definidora de los rumbos de la integración, en su aspecto relacional (identificado por Hurrell con el término cooperación); y al legitimar el accionar colectivo a partir de la construcción conjunta de mecanismos de gobernanza (cohesión) regional que no supongan consecución de etapas, sino gradualidad. Así, se piensa en una dimensión relacional, con más de un grado de interacción, cooperación, coordinación e integración entre los actores.

Malamud (2011, p. 220) recuperó las palabras de Ernst Haas al definir a la integración como un proceso a través del cual los Estados «se mezclan, se confunden y se funden voluntariamente con sus vecinos de modo tal que pierden ciertos atributos de soberanía, para adquirir, al mismo tiempo, nuevas técnicas de resolución conjunta de conflictos». El autor considera que los procesos de integración deben ser necesariamente conducidos desde el Estado, atribuyéndole a la política el rol de articulación de intereses comunes. Resalta, además, la dimensión de la legitimidad y la deliberación de la comunidad política cuando existen este tipo de iniciativas y enfatiza su pretensión de construcción estatal. Sin embargo, al diferenciar la integración de la gobernanza regional, que correspondería a los «mecanismos a través de los cuales se regulan las nuevas comunidades» (Malamud, 2011, p. 223), restringe a actores sociales su construcción y centra su análisis a la cuestión de la delegación de soberanía. De hecho, hay acepciones terminológicas antecedentes a nuestro esquema analítico que son valiosas en sus formulaciones argumentales, pero no tanto en su cuidado conceptual, que entendemos comple- 
mentarias y que, muchas veces, contraponen conceptos entre sí sin ser necesario. La gobernanza, entendida como construcción colectiva, es dependiente de la trayectoria y de los costos que traen los cambios de conducción política (Lima y Milani, 2016, p. 23); pero no necesariamente debemos entenderla como opuesta a la cooperación política o a la integración económico-comercial (tal como los autores sugieren, p. 24). La gobernanza tiene necesariamente legitimidad deliberativa al estar construida desde abajo (actores sociales de la integración) y desde arriba (por gobiernos representativos). Esta vinculación entre la construcción estatal y societal de la gobernanza es lo que pretendemos recuperar, su concepción pública.

La concepción presentada por Hurrell (1995) del término regionalización atiende parcialmente la dinámica sudamericana por identificarla apenas como un proceso botton-up entre actores prioritariamente no estatales. En este artículo se entiende que la regionalización abarca diferentes procesos de interacción entre actores económicos y sociales de un área territorial específica que, consecuentemente, se identifica con una determinada región normativamente construida, y que pueden o no tener incentivos directos por parte de los gobiernos. En ese sentido, el término regionalización utilizado en su acepción tradicional (Hettne y Söderbaum, 2000) para explicar los procesos en que los actores cooperan, se complementan y convergen a través de espacios geográficos que, en general, son transnacionales, aunque pueden llegar a abarcar subregiones de un Estado; se complementa con la característica principal que aquí se pretende señalar como resultado de la construcción de gobernanza sudamericana: los incentivos para la reproducción de marcos colectivos de acción, sean estos promovidos desde ámbitos gubernamentales, o no; tanto desde marcos institucionales ya constituidos o de nuevas prácticas. Tal proceso reproduce más regionalización y genera demandas por construcción de gobernanza.

Pía Riggirozzi (2010) propuso estudiar los aspectos sociopolíticos e institucionales de la construcción regional latinoamericana conjuntamente a partir del término propuesto por Hettne y Söderbaum (2000), regionalidad. Herramienta analítica que estudia los resultados de la regionalización en sus múltiples dimensiones, ideológicas y prácticas. Además, permite separar la cuestión ontológica de la trayectoria específica que el regionalismo adopte, va más allá de una concepción estadocéntrica $y$, fundamentalmente, porque coloca a la idea de la regionalidad en transformación y construcción constante por parte de tales actores. Aunque se considera necesaria su complementariedad con otros abordajes menos lineales en su fundamentación básica, como los que reclaman el protagonismo político de la región (Riggirozzi y Tussie, 2017) y la centralidad de esta última para reclamar espacios globales de acción (Acharya, 2007). Ambos 
términos, regionalidad y regionalización han sido importantes en los desarrollos conceptuales del regionalismo sudamericano. En ese sentido, son dimensiones del concepto de gobernanza regional relevantes para entender también su carácter dinámico y diacrónico.

Por otro lado, la dimensión geográfica del concepto de región tiene implicaciones territoriales, culturales y normativas; la contigüidad territorial refuerza la tendencia a compartir valores y costumbres; la condición de vecino sería un adicional que generaría por sí misma cierto nivel de interacción, cooperación, coordinación e integración entre territorios, antes que países, lo que implicaría, también, su condición de frontera. Así, cabrían implicaciones socioeconómicas de hecho, a las construcciones políticas. Estos procesos se darían más entre actores privados (incentivos para cooperar entre cadenas productivas o mercados complementarios y ampliados); pero también permearían ámbitos públicos al expandir y reproducir al capitalismo nacional (Amin, 2008).

También ha habido acumulación a largo y mediano plazo en las prácticas y experiencias sudamericanas de integración; en especial, en los micro procesos y sus múltiples niveles de toma de decisiones y ejecución. Podemos identificar algunas características que se reproducen y que podrían dar lugar a patrones y mecanismos de resiliencia cíclicos. A partir de cambios estratégicos en la conducción ideológica, es posible pensar en stop's and go's de modelos o proyectos de integración y en estrategias más o menos pragmáticas de acción a nivel político que dejan interrogantes sobre el devenir de la integración (Briceño-Ruiz, 2018).

En ese sentido, se entiende que la incertidumbre y la heterogeneidad han sido características claves del regionalismo, algunos expertos llegaron tempranamente a la misma conclusión respecto al carácter abierto de las experiencias latinoamericanas (Saraiva, 2011; Caetano, 2011; Solanas, 2011); sería importante también evaluar si tal heterogeneidad no es consecuencia de ciertos niveles de pragmatismo en la ejecución del ideario integracionista regional. La idea de solapamiento, flexibilidad, ineficacia, o la ya tradicional crítica a la intergubernamentalidad (Caetano, 2011) cambian de perspectiva y pasan a ser vistas como resultados del pragmatismo y de la eficiencia en la construcción de los acuerdos. Esta dimensión relacional es reivindicada por Dabène (2009, 2012) quien a partir de la idea de Moravcsik $(1997 ; 1998)$ de congelamiento de preferencias a través del tiempo mediante mecanismos de lock in de las decisiones tomadas en acuerdos identifica ejemplos de resiliencia o supervivencia de consensos en el ámbito latinoamericano.

¿Cuál es la autonomía que las diferentes experiencias de integración tienen respecto a las condiciones en que fueron creadas? Las respuestas requerirían 
estudios empíricos minuciosos para identificar tales aspectos y considerarlos además de como ejemplos de resistencia o resiliencia- como posibles modelos de interacción-cooperación-integración regional sudamericano. El modelo conceptual planteado en este trabajo puede ayudar a entender varios de esos patrones a partir de sus trayectorias; se piensa en la gobernanza regional y en sus diferentes construcciones interpretativas por medio de los autores que han trabajado tal concepto para, posteriormente, delinear la propuesta argumental.

\subsection{Conceptualización de la gobernanza regional y nuevas dimensiones transversales}

Ambos términos, utilizados conjunta o separadamente, gobernanza y regional, remiten varias acepciones en su significado cotidiano; sin embargo, existen abordajes que son considerados antecedentes de la propuesta que se presenta.

Nolte (2011), se refiere a la gobernanza regional como un conjunto de organizaciones, principios y reglas normativas del comportamiento estatal, así como al proceso de creación de tales organizaciones y principios que contribuirían a la solución de problemas comunes, proporcionando beneficios en las relaciones intrarregionales. Nolte (2013) subraya el concepto de gobernanza regional como un instrumento analítico para la comprensión del regionalismo en América Latina; sugiere la sustitución de la tradicional división entre integración y cooperación estatal para pensar el regionalismo latinoamericano por la adopción de la arquitectura de la gobernanza regional, para dar cuenta de la proliferación y solapamiento de los diferentes proyectos de integración sin ser deterministas de su ineficacia ex ante (Nolte, 2014, p. 5).

La propuesta aquí expuesta pretende complementar tal iniciativa al adicionar a la idea de gobernanza la posibilidad de que los actores implicados (amplios, no tradicionales) asuman su accionar colectivo en torno a un continuo interacción-cooperación-coordinación-integración que traspasa transversalmente las dos dimensiones delineadas durante la discusión conceptual anterior: la regionalidad y la convergencia/divergencia ideológica sobre los diferentes proyectos que dieron lugar al establecimiento de distintos patrones de regionalismo a lo largo de la historia.

Conceptualmente las dimensiones de regionalidad e ideología se pueden articular en ese continuo de relaciones entre los actores que interactúan, cooperan, coordinan y se integran, sean actores con legítima y tradicional competencia para ello (gobiernos, agencias estatales) o que, al asumir la idea de regionalidad transversal, adquieren protagonismo en otros escenarios de disputa de intereses (empresarios, ciudadanos, transnacionales regionales, colectivos regionales). 
La regionalización planteada anteriormente es importante en la comprensión de los microprocesos que necesitan de la gobernanza regional; la integración económico-comercial que existe formal o informalmente en regiones transfronterizas redunda en cierta regionalización de procesos productivos, dinámicas propias desarrolladas en las fronteras y prácticas institucionales específicas planteadas con diferentes niveles de coordinación o cooperación a nivel institucional —estatutos y proyectos de gestión de fronteras que han sido ensayados a nivel regional. Se destaca de la noción de necesidad de cierta gobernanza: estos procesos contribuyen al desarrollo de demandas de gobernanza regional autosostenidas en el tiempo (lo que trae implícito el posicionamiento en algún lugar del continuo interacción-cooperación-coordinación-integración).

Otro de los aspectos que tal gobernanza regional debe tener enfatizado es su dimensión pública. La provisión de bienes públicos a nivel regional pasa necesariamente por una gobernanza compartida. En ese sentido, la noción de regionalismo regulatorio (Jayasuriya, 2009) brinda las condiciones para que los esfuerzos integradores que se materialicen en bienes públicos regionales puedan tener un sustento institucional y sean, por tanto, más que la política externa del regional paymaster de turno (Mattli, 1999). La persistencia en el tiempo de tales demandas y el establecimiento de sus reglas y reglamentos coordinados en el marco de la gobernanza regional son de carácter público y colectivo. La multiplicación de asuntos tratados dentro de los espacios regionales y la institucionalización de la participación de actores no tradicionales que toman tales decisiones podrían derivar en la creación de incentivos para generar políticas públicas de nivel regional. Sin embargo, siempre se encuentra la limitación de la convergencia ideológica intergubernamental como elemento necesario para la aplicación de la gobernanza regional en momentos más comprometidos del continuo, por ejemplo, en la coordinación y ejecución de los planes y proyectos ya creados.

La acepción de gobernanza regional en tanto concepto que engloba también la formulación y gestión de políticas públicas regionales fue propuesta por Lujan (2009), quien advertía su condición pública, común o colectiva y esgrimía algunas dimensiones para el análisis de su aplicabilidad. Asimismo, la connotación de gobernanza como «la capacidad que tienen las sociedades humanas de proveerse sistemas de representación, instituciones, procesos, cuerpos sociales, para administrarse ellas mismas en un movimiento voluntario» (Calamé y Talmant, 2001, p. 15) también trae a colación esta cuestión pública de la gobernanza y su legitimidad en tanto acción colectiva.

Estos autores asimilan gobernanza regional a las nociones que se quieren enfatizar: el carácter necesario para la gestión de zonas, recursos e interacciones comunes; y la condición de pública y legítima, en tanto discutida dentro de térmi- 
nos democráticos participativos y representativos; ambas cuestiones que también determinarían y ameritarían algún tipo de cooperación, coordinación o integración política a nivel regional.

La contribución aquí presentada al concepto de gobernanza regional está directamente vinculada a las dimensiones transversales al continuo de interacción-cooperación-coordinación-integración en los que se coloca a los actores relevantes (estatales, subregionales, públicos y privados). En ese sentido, tanto la noción de regionalización en su aspecto más geográfico, como la de regionalidad - conjugados los demás elementos identitarios y discursivos - son aspectos intrínsecos a la construcción conjunta de una gobernanza regional que ya no solo es un objetivo ideológico político, sino también necesario de la vida en esos contextos. Por otra parte, la convergencia o divergencia ideológica —en su aspecto diacrónico - le atribuyen al concepto de gobernanza regional cierto elemento contextual que es igualmente relevante para el análisis, puesto que permite comprender su dinámica y variaciones. También en esta dimensión, algún tipo de construcción de gobernanza regional en torno al continuo interacción-cooperación-coordinación-integración es necesario, puede ser constante, variable o el piso mínimo para actuar conjuntamente. 
Figura 1: Representación del esquema analítico de la gobernanza regional

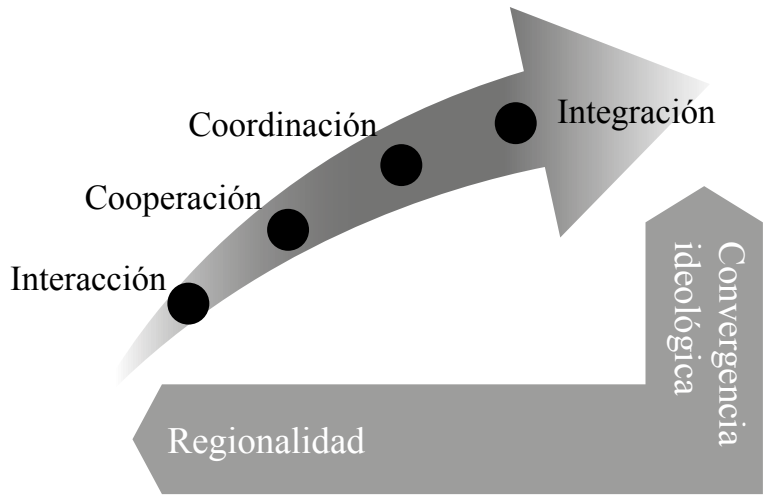

Fuente: Elaboración propia.

Los actores de la gobernanza regional se posicionan en un continuo que tiene dos características que se han modelado como transversales, ya que aparecen en todos los grados de entendimiento representados: la regionalidad y la convergencia/divergencia ideológica; ambas variables dinámicas y plausibles de ser graduadas. Tal representación gráfica permite analizar los diferentes proyectos de integración latinoamericana (regionalismos) sin limitaciones conceptualmente preestablecidas o de juicios valorativos respecto a su deseabilidad o eficiencia; asimismo, las dos características transversales que se introducen en el análisis permiten la contextualización de los ejemplos abordados.

En resumen, se propone que la gobernanza regional (figura 1), en su dimensión activa conjunta o comunitaria, se puede representar en un continuo en el que hay gradualmente niveles diferentes de interacción, cooperación, coordinación o integración entre los distintos actores. En un extremo, las partes apenas tendrían una interacción común, inclusive conflictiva; en el otro extremo, las partes cooperarían en torno a ciertas agendas previamente establecidas (definidas ideológicamente). Con la coordinación se pretende tener una posición común, conjunta sobre el/los asuntos en cuestión, se estaría en ese sentido, un poco más hacia el otro lado del continuo; finalmente, en el otro extremo, la integración implicaría acciones y planificación coordinada en forma conjunta, lo que, necesariamente, llevaría al establecimiento de algún tipo de institucionalidad para la consecución de sus objetivos que, de perpetuarse, establecería políticas públicas regionales. A lo largo del continuo se dibujan diferentes términos y acuerdos de planificación estratégica que implican mayor o menor capacidad de negociación y ejecución conjunta, todos los puntos del continuo representan determinada gobernanza regional. 
Los diferentes grados de entendimiento entre los actores de la integración (no solamente países o gobiernos) son dependientes de las dos dimensiones transversales, que también son de carácter dinámico y evolutivo. La dimensión regionalidad, que tiene un atributo espacial geográfico y otro construido; y la dimensión ideológica, de carácter netamente político. Asimismo, la dimensión ideológica, tiene dos aspectos interesantes: la posible convergencia entre los diferentes proyectos de integración que cada país/gobierno considere oportuno, y sus propios efectos prácticos que no necesariamente son convergentes ideológicamente, sino que, más bien, han tenido siempre limitaciones pragmáticas. Así, la gobernanza regional es un concepto amplio en su dimensión ideológica. A continuación, se realiza un esfuerzo por identificar tales patrones en los marcos de las hipótesis planteadas por este artículo.

\section{Patrones de convergencia/divergencia ideológica en los regionalismos sudamericanos}

El mayor o menor entendimiento entre los actores regionales latinoamericanos en el continuo que se ha representado anteriormente ha derivado en la construcción desde los gobiernos (top-down) de diferentes proyectos con varios grados de planificación coordinada estratégicamente. En tales instancias, la configuración regional adoptada está determinada por la mayor o menor convergencia/divergencia político-ideológica entre los gobiernos que les dieron impulso, lo que ha redundado en distintos escenarios regionales. En ese sentido, la convergencia ideológica en torno al proyecto integracionista a construir (gobernanza) es una condición suficiente para su consecución. Una revisión de los análisis de expertos arroja algunos consensos: han denominado regionalismo o, en plural, regionalis$m o s$, a las diferentes etapas y han adjetivado a tal término con algún otro concepto que los caracteriza. No se pretende hacer aquí un recorrido histórico de las iniciativas de integración, sino identificar patrones de convergencia/divergencia que se plantearon más claramente en la trayectoria contemporánea sudamericana a fin de ilustrar el argumento.

Así, hubo alta convergencia ideológica durante la redemocratización de la segunda mitad de la década de 1980 en torno a la integración comercial y unidireccional alcanzada, incluso como reacción a la globalización (Mattli, 1999), se creía que la integración de mercados era la condición para la inserción internacional de la región, o para la consolidación de los nuevos regímenes democráticos en el caso del Cono Sur (Lima y Coutinho, 2007). Tal convergencia derivó en lo que la literatura ha llamado regionalismo abierto, que tenía como base fun- 
damental los preceptos de apertura de mercados y liberalismo comercial. ${ }^{l}$ En su primera década de convergencia liberal los proyectos integracionistas de la época avanzaron en la negociación conjunta de aranceles comunes, liberalizando áreas donde el comercio producía mayores ganancias y desarrollando el comercio intrarregional. Sin embargo, este proceso se vio interrumpido por sus magros resultados en términos de desarrollo social y económico; la desvalorización cambiaria brasileña interrumpió la creación de comercio iniciada en la década de 1990 y rápidamente se vieron las consecuencias de las reacciones pragmáticas y descoordinadas. La idea de crear una cadena de producción en escala entre socios de la región nunca llegó a ser implementada debido a la resistencia de actores económicos domésticos que colocaron obstáculos a las iniciativas de carácter defensivo de los procesos de integración regionales. En el pasaje de siglo algunas de las condiciones básicas para el funcionamiento del regionalismo abierto se modificaron.

Se abre un período de divergencia ideológica en torno al modelo de integración deseable o presumible. La denominada integración ampliada fue parte del discurso de los actores en ese período de divergencia; entendida como la ampliación de los esfuerzos de coordinación y cooperación políticas hacia otras dimensiones: social, productiva, infraestructural, etc. (Sarti, Leite, Perrotta, y Cardoso, 2012). Sin embargo, y a falta de consenso sobre cuáles serían las características distintivas de los nuevos proyectos en términos prácticos, no se ensayaron nuevas terminologías para definirlo en sus aspectos intrínsecos, a excepción de algunos aportes que tuvieron al eje político ideológico como principal dimensión considerada en el análisis del regionalismo latinoamericano (Dabéne, 2009).

Paulatinamente, comenzaron a establecerse mecanismos de protección de mercados que, al cambiar el signo ideológico de los gobiernos de la región, se convirtieron, abiertamente, en políticas de desarrollo nacional de corte proteccionista o neodesarrollista. Estableciéndose, entonces, un nuevo período de convergencia ideológica denominado consenso progresista. ${ }^{2}$

Junto al objetivo básico de reducción de la pobreza y de la desigualdad, los nuevos gobiernos convergieron en sus políticas externas mediante la adopción de tendencias a la negociación colectiva de asuntos múltiples, estableciendo preferencias cooperativas a nivel regional que determinaron más construcción de gobernanza y el cambio hacia un nuevo regionalismo. Uno de los términos más

1 Briceño-Ruiz (2018) identifica tres modelos de integración económica y tres de cooperación política, en su concepción, el regionalismo de esa época debería denominarse regionalismo estratégico.

2 También este término ha sido muy debatido en la literatura, pensando en la mejor manera de etiquetar al período: gobiernos progresistas (Lima, M. (Org.), 2008); marea rosa (Panizza, 2006; 2009; Silva, 2009, 2010). 
utilizados para referirse a este período es el de regionalismo posliberal; sugerido inicialmente en un trabajo publicado por la Cepal (Veiga y Rios, 2007). La característica fundamental y distintiva de esa nueva configuración de la integración es, según los autores, la apertura hacia aspectos políticos como asuntos relevantes a coordinar entre los países de la región; por sobre el protagonismo que la integración comercial tuviera otrora. Ese nuevo espacio de la política trae a la agenda común la discusión sobre las asimetrías regionales, los problemas de integración física, la necesidad de establecer posicionamientos comunes frente a ámbitos multilaterales globales, entre otros asuntos políticos que adquieren relevancia. El regionalismo supera a la vertiente ideológica liberal que caracterizara las negociaciones de la década anterior; para reposicionarse en torno a otra convergencia. El Foro de San Pablo, al congregar a partidos de izquierda de la región, fue local de debate de esas proposiciones (Dabène, 2012b).

Al de Veiga y Rios se sumaron análisis como el de Sanahuja (2008, 2010, 2012), que destaca del regionalismo posliberal su orientación a la construcción de estados desarrollistas, en oposición a la integración como instrumento de inserción internacional en un mundo globalizado, característica del modelo anterior. Esta concepción enfatiza la revitalización del papel del Estado en la construcción social y política de la integración con una perspectiva autonomista. Los gobiernos de la región tendrían como objetivo superar el trilema entre la estrategia de desarrollo autonómico, la defensa de la soberanía nacional y la autonomía en el plano internacional (Sanahuja 2012, p. 22), por tales motivos el regionalismo construido por esa época ha sido denominado de productivo (Briceño-Ruiz, 2018). En ese sentido, había alta convergencia en torno a la idea de integración como instrumento de desarrollo, pero disminuía al pensar en su ejecución habiendo variados ejemplos de conductas pragmáticas en tal instancia.

Riggirozzi y Tussie (2012) adoptaron el término regionalismo poshegemónico para enfatizar el carácter estructural de las nuevas propuestas en dos sentidos: respecto a la nueva capacidad desarrollista-autónoma que intentaron construir y con relación a la posición que Latinoamérica asumió en el escenario global frente al liderazgo estadounidense. Se estaría recuperando la idea de regionalismo defensivo en un sentido ideológico-político, profundamente crítico de la etapa anterior neoliberal y orientado para el desarrollo humano en una perspectiva más amplia de integración (p.139). Ya, Briceño-Ruiz y Ribeiro Hoffmann (2015) apuntan al regionalismo poshegemónico enfatizando su pluralidad, con la coexistencia y sobreposición de diferentes modelos de cooperación e integración. En tal sentido, la coexistencia de diferentes proyectos denota la no existencia de un consenso hegemónico de un tipo de regionalismo y abre la posibilidad para la construcción de modelos antagónicos (Briceño-Ruiz, 2017b). 
La construcción de la trayectoria histórica del regionalismo latinoamericano realizada por Dabène (2012b), quien denominaba a las diferentes olas de regionalismo como ciclos de politización en los que las preferencias políticas de sus arquitectos - institucionalizados en la figura de los presidentes, cuyas acciones nombra como presidencialismo colectivo - estarían demostrando el mayor o menor énfasis en los aspectos políticos de la integración, abre un precedente ante la posibilidad de existir conflictos desde la perspectiva de la divergencia ideológica entre los diferentes actores. Dabène llamaba la atención para las posibles tensiones ante la multiplicidad de actores y de agendas que proliferan dentro de los espacios institucionales. Por su parte, Gardini (2015) proponía el término regionalismo modular, destacando el crecimiento de las iniciativas yuxtapuestas, y la distancia entre las narrativas de los actores políticos de la realidad (o sea, la retórica); así como criticaba las experiencias de cooperación en detrimento de las iniciativas de integración y proponía la superación del regionalismo abierto como instrumento analítico (p. 220). Sin embargo, delante de la encrucijada política actual, y frente a la creciente complejidad inherente (Gómez-Mera, 2015) es posible que se reivindique al regionalismo abierto como una nueva convergencia o hegemonía, este último aspecto es levantado por Briceño-Ruiz-Ruiz (2017a y $2017 b)$.

No obstante, así como en la agenda de la integración económico-comercial se colaron aspectos de otras agendas que también merecieron interacciones, cooperación y coordinación, como la seguridad, la educación o la movilidad; en el período de convergencia progresista, también se realizaron esfuerzos tendientes a alcanzar acuerdos económico-comerciales. Esta agenda, al contrario de lo que podría pensarse, no solo fue activa, sino también fue efectiva. La integración comercial de los 90 llevó a demandas por más y mejor integración, provenientes de sectores políticos y sociales; también obtuvo resultados importantes en su dimensión principal (la inserción comercial). Asimismo, a pesar del énfasis integracionista en la ampliación a otras dimensiones como la social, productiva (no meramente comercial) y representativo-participativa durante el consenso progresista; la dimensión económico comercial de la integración se vio igualmente beneficiada.

Los argumentos que han sido desarrollados ilustran de manera muy elocuente como han sido las principales experiencias del regionalismo en sus características básicas; sin embargo, las polarizaciones y los adjetivos no rescatan la variedad de tonalidades que existen entre uno y otro extremo. Pueden considerarse ciertas características compartidas tanto por las experiencias de integración ensayadas, como por los analistas al estudiar ese período específico en que los gobiernos progresistas o bien innovaron institucionalmente o se adueñaron de los proyectos 
de integración existentes (creando nuevas institucionalidades en las viejas estructuras) y construyeron nuevas estrategias de acción conjunta a nivel regional. Ambas cosas constituyeron una manera de construir la gobernanza regional diferente de las experimentadas antes.

A los proyectos de integración de la fase anterior, Mercosur y CAN, se les sumaron, en la primera década del siglo XXI la Unasur, la Alianza Bolivariana para los Pueblos de Nuestra Américas (ALBA) y, en la segunda década, la Comunidad de Estados Latinoamericanos y Caribeños (Celac). A excepción de la CAN, que se vio sumamente debilitada durante el período, todas las demás experiencias incorporaron amplias agendas de cooperación regional. A su vez, estas nuevas experiencias regionales aseguraron la autonomía de los Estados miembro en sus respectivas estrategias de desarrollo, teniendo a la política como principal eje articulador de los diferentes consensos necesarios para construir la integración más allá del comercio.

Sin embargo, a partir de la segunda década del siglo algunos acontecimientos fueron modificando el escenario regional, cambió la convergencia ideológica, pasando por un nuevo período de divergencia en los proyectos integracionistas. La crisis económica internacional iniciada en 2008, que no había tenido consecuencias inmediatas, trajo cambios en la economía internacional que acabaron impactando posteriormente. La caída de los precios de los commodities exportados por los países de la región impactó en las economías nacionales desacelerando el crecimiento que habían experimentado durante la primera década; los gobiernos progresistas vieron debilitadas sus capacidades de implementar políticas públicas de crecimiento económico y redistribución social conjuntamente, redundando en crisis económicas. Estos escenarios, por su parte, trajeron consecuencias en los dos niveles, al impacto de la crisis económica se les sumaron problemas de gestión y crisis políticas. Ambas cuestiones favorecieron el cambio en el perfil político de la región, emergieron gobiernos con perfiles liberales y conservadores en oposición a los anteriores gobiernos progresistas, modificándose así el proyecto político que ve a la integración como un instrumento para el desarrollo y, consecuentemente, sus prácticas.

Se inaugura así, otro período de divergencia o de coexistencia de dos proyectos de integración (Briceño-Ruiz, 2017a, 2018). En 2011 se conformó la Alianza del Pacífico que aglutinó países asociados al Mercosur, cuyas economías se orientan básicamente a la apertura comercial exterior y cuyos gobiernos no convergían ideológicamente con el subrregionalismo del sur, lo que la literatura ha denominado de división Atlántico-Pacífico (Briceño-Ruiz y Morales, 2017). Esto trajo nuevamente a la agenda el debate sobre la recuperación del regionalismo abierto de la década de 1990, así como dejó en evidencia la división existente en- 
tre las comunidades epistémicas prointegracionistas de la región, habría entonces dos visiones diferentes de regionalismo: una liberal-comercial y otra política-integradora, ambas con instrumentos específicos de coordinación de intereses y con diferentes resultados en términos de éxito o fracaso de sus iniciativas. Cambios y transformaciones propias de los regímenes democráticos a nivel doméstico pueden modificar las preferencias nacionales respecto a qué tipo de regionalismo es deseado o necesario, y eso afecta directamente a las instituciones ya consolidadas (Sanahuja, 2017).

El desmonte progresivo (o la indiferencia) de los mecanismos de cooperación, coordinación e integración que fueron producto del regionalismo posliberal (o poshegemónico), amplió el arco de propuestas en torno a otras maneras de entender la integración, reforzando la ausencia de un modelo de regionalismo predominante (o hegemónico); las divergencias ideológicas promueven la coexistencia de diferentes modelos a través de solapamientos o yuxtaposiciones dada la necesidad de algún tipo de gobernanza. En ese sentido, la idea de continuo que se intenta delinear como marco analítico puede arrojar luz sobre las diferentes maneras en que la gobernanza regional adquiere sus formas.

\section{Reflexiones finales}

En los últimos tiempos ha habido una reconfiguración de la acción colectiva a nivel regional, con cambios en sus estructuras institucionales y en sus canales de acción que se han visto modificados en varias formas; tal vez la suspensión de la participación en la Unasur por parte de los seis países con gobiernos conservadores y el riesgo real de terminar con la organización sea el mejor ejemplo de ello, aunque el movimiento colectivo evidencia también cierto grado de coordinación intergubernamental. Los desdoblamientos de la crisis venezolana podrán incluso generar nuevas hipótesis en ese sentido.

A lo largo del tiempo se encuentran patrones del regionalismo autonómico (década de 1960) recreados en el momento posliberal; así como características del regionalismo abierto recreadas en la actualidad; todas ellas con una flexibilidad no imaginada; lo que coloca al pragmatismo como elemento distintivo del regionalismo también de una manera recurrente. En ese sentido, la recreación de patrones de convergencia y divergencia ideológica cíclicamente daría lugar, paulatinamente, a que la gobernanza regional se vea cada vez más comprometida en ese eje que iría entre la ideología y el pragmatismo, enmarcado por el continuo de consensos negociados y por la construcción institucional con diferentes grados de interacción-cooperación-coordinación-integración. El momento político actual, con la elección de diferentes presidentes de orientación liberal y, en algunos 
casos, articulados con conservadurismo de costumbres y trazos de nacionalismo, trae a la luz interrogantes sobre la situación y el futuro próximo de la idea de integración regional.

Así, la integración sudamericana se encuentra en una encrucijada con caminos y posibilidades difusas, solapadas y con grados de dificultad elevada. En cualquier nivel del continuo que se encuentre la dimensión relacional, cierto grado de cooperación económico-comercial siempre habrá en la región; tendrá que haber, también, al menos, cierta coordinación entre los mercados, que estará vinculada a determinado grado de cooperación política; no pueden ir separados. Para ello, la convergencia ideológica en torno a la práctica de la integración y sus usos se hace una condición suficiente para la construcción conjunta. Sin embargo, existen momentos en que ciertos niveles de pragmatismo son identificados como necesarios. Actualmente, se atiende a la convivencia de dos diferentes modelos 0 ideas de integración que no convergen. En ese sentido, y pensando en una alternativa desde la praxis, a partir de la necesidad de gestionar recursos y políticas públicas comunes para problemas también comunes estaría dada la motivación principal para la construcción de mecanismos de gobernanza regional; cuando la convergencia política no existe o es mínima, cabría la necesidad de organizar la acción colectiva de alguna manera a partir de elementos ya construidos en períodos anteriores o de demandas societal y estructuralmente impuestas. Preguntarse cómo se configuran esas demandas y sus respectivos efectos a nivel político constituyen nuevas interrogaciones para una agenda de investigación futura.

Aquí se adelantan ciertos elementos que tal agenda debería incorporar en su estrategia metodológica. Además de la utilización de la formulación conceptual propuesta, este trabajo plantea a la perspectiva de larga duración como base de la formulación argumental, por lo que una agenda de investigación futura necesariamente partiría del análisis de la trayectoria. Por otro lado, la utilización de herramientas metodológicas múltiples para dar cuenta de los resultados observados en los dos grandes momentos convergentes ideológicamente que fueron expuestos es, también, fundamental en la construcción de los argumentos. En primer lugar, sería necesario pensar en los ejemplos pragmáticos que se han sucedido a lo largo del tiempo en los dos momentos de alta convergencia ideológica en torno a uno de los dos modelos o ideas proyecto de integración que se delinearon. En segundo lugar, sería importante ver cómo se sigue construyendo la gobernanza regional sudamericana a lo largo del continuo y pensar en cómo la creciente regionalidad podría abrir caminos para una cierta autonomía de la convergencia política. 


\section{Referencias}

Acharya, A. (2007). The Emerging Regional Architecture of World Politics. World Politics, 59(4), 629-652.

Amin, A. (2008). Regiones sin fronteras: hacia una nueva política del lugar. En V. Fernández, A. Amin, y J. I. Vigil (Comp.), Repensando el Desarrollo Regional, contribuciones globales para una estrategia latinoamericana. Buenos Aires: Argentina: Miño y Dávila.

Bizzozero, L. (2011). América Latina a inicios de la segunda década del siglo xxi: entre el regionalismo estratégico y la regionalización fragmentada. Revista Brasileira de Política Internacional, 54(1), 29-43.

BricEÑo-Ruiz, J., y Rivarola, A. (2013). The resilience of regionalism in Latin American and the Caribbean. Londres, Reino Unido: Palgrave Macmillan UK.

Briceño-Ruiz, J., y Ribeiro Hoffmann, A. (2015). Post-hegemonic regionalism, Unasur and the reconfiguration of regional cooperation in South America. Canadian Journal of Latin America and Caribbean Studies, 40(1), 48-62.

Briceño-Ruiz, J., y Morales, I (Eds.) (2017). Post-Hegemonic Regionalism in the Americas: Toward a Pacific-Atlantic Divide? Londres y Nueva York, Reino Unido y EE. UU.: Routledge.

Briceño-Ruiz, J. (2017a). ¿Un nuevo ciclo regionalista en América Latina? Debates conceptuales, modelos y realidades. Cuadernos Americanos, 3(161), 15-45.

Briceño-Ruiz, J. (2017b). Latin America beyond the continental divide. Open Regionalism and Post-hegemonic regionalism co-existence in a changing region. En J. Briceño-Ruiz, I. Morales (Eds.), Post-Hegemonic Regionalism in the Americas: Toward a Pacific-Atlantic Divide? (pp. 73-98). Londres y Nueva York, Reino Unido y EE. UU.: Routledge.

Briceño-Ruiz, J. (2018). Times of Change in Latin American Regionalism. Contexto Internacional, 40(3), 573-594.

Caetano, G. (Coord.) (2011). Mercosur 20 años. Montevideo; Uruguay: CEFIR.

Calamé, P., y Talmant, A. (2001). Con el Estado en el Corazón. El andamiaje de la gobernancia. Montevideo, Uruguay: TRILCE.

Cienfuegos, M., y Sanahuja, J. A. (2010). Una región en construcción. Barcelona, España: Fundació CIDOB.

DABÈne, O. (2009). The Politics of Regional Integration in Latin América. Theoretical and Comparative Explorations. Nueva York, EE. UU.: Palgrave Macmillan UK. 
DabÈne, O. (2012a). Consistency and Resilience Through Cycles of Repolitization. En P. Riggirozzi, y D. Tussie, The Rise of Post-hegemonic Regionalism (pp. 41-64). Londres, Reino Unido: Springer.

DABÈne, O. (2012b). Explaining Latin America's fourth wave of regionalism. Regional integration of a third kind. Artículo presentado en el Congreso Latinoamericano de Estudios Sociales (LASA), San Francisco, EE. UU.

FAWCETT, L. (2013). The history and concept of Regionalism. UNU-CRIS Working Papers W-2013/5. Brujas, Bélgica: UNU-CRIS.

GARDInI, G. L. (2011). Latin American Foreign Policies between Ideology and Pragmatism: a framework for analyses. En G. L. Gardini, y P. Lambert, Latin America Foreign Policies between Ideology and Pragmatism. New York, EE. UU.: Palgrave Macmillan UK.

GARDINI, G. L. (2015). Towards modular regionalism: the proliferation of Latin American cooperation. Revista Brasileira de Politica Internacional, 58(1), 210-229.

Gómez-Mera, L. (2015). International Regime Complexity and Regional Governance: Evidence from the Americas. Global Governance, 21(1), 19-42.

Hettne, B., y Söderbaum, F. (2000). Theorising the Rise of Regionness. New Political Economy, 5(3), 457-472.

Hurrell, A. (1995). O ressurgimento do regionalismo na política mundial. Contexto Internacional, 17(1), 23-44.

JAYASURIYA, K. (2009). Regulatory regionalism in the Asia-Pacific: drivers, instruments and actors. Australian Journal of International Affairs, 63(3), 335347.

Lima, M. R. y Coutinho, M. V. (2007). Agenda Sul-Americana: Mudanças e desafios no início do século XXI. Brasilia; Brasil: Fundação Alexandre Gusmão.

Lima, M. R. y Coutinho, M. V. (Org.). (2008). Desempenho dos Governos Progressistas do Cone Sul: Agendas Alternativas al Neoliberalismo. Rio de Janeiro, Brasil: IUPERJ.

Lima, M. R. y Milani, C. R. S. (2016). Política externa, geopolítica e modelos de desenvolvimento. En M. R. Soares de Lima; C. R. S. Milani; E. Echart Muñoz (Eds.), Cooperación Sur-Sur, política exterior y modelos de desarrollo en América Latina. Buenos Aires, Argentina: CLACSO-FAPERJ.

LuJAN, C. (2009). Una reflexión sobre los soportes institucionales para viabilizar políticas públicas regionales. [CD Rom] La reforma Institucional del Mercosur. 
Del diagnóstico a las propuestas (pp. 183-197). Montevideo, Uruguay: Trilce.

Malamud, A. (2003). Presidentialism and Mercosur: A Hidden Cause for a Successful Experience. En F. Laursen, Comparative Regional Integration: Theoretical Perspectives (pp. 53-73). Aldershot, Reino Unido: Ashgate.

Malamud, A. (2011). Conceptos, teorías y debates sobre la integración regional. Norteamérica, 6(2): 219-249.

Malamud, A. y Gardini, G. L. (2012). Has Regionalism Peaked? The Latin American Quagmire and its Lessons. The International Spectator: Italian Journal of International Affairs, 47(1), 116-133.

Mattli, W. (1999). The Logic of Regional Integration. Europe and Beyond. Cambridge, Reino Unido: Cambridge University Press.

Moravcsik, A. (1997). Taking Preferences Seriously: A Liberal Theory of International Politics. International Organization, 51(4), 513-553.

Moravcsik, A. (1998). The Choice for Europe. Social Purpose and State Power from Messina to Maastricht. New York, EE. UU.: Cornell University Press.

Nolte, D. (2011). Regional powers and regional governance. En D. Nabers, y D. Godehardt (Eds.), Regional powers and regional orders (pp. 49-67). Londres y Nueva York, Reino Unido y EE. UU.: Routledge.

Nolte, D. (29 de mayo - 1 de junio de 2013). Latin America's New Regional Architecture: Segmented Regionalism or Cooperative Regional Governance? XXXI International Congress of the Latin American Studies Association (LASA). Congreso llevado a cabo en Washington D.C., EE. UU

Nolte, D. (2014). Latin America's New Regional Architecture: A Cooperative or Segmented Regional Governance Complex? EUI Working Paper RSCAS 2014/89. Fiesole, Italia: European University Institute. Robert Schuman Centre for Advanced Studies.

Nolte, D., y Comini, N. M. (2016). Unasur: Regional Pluralism as a strategic outcome. Contexto Internacional, 38(2), 545-565.

Panizza, F. (2006). La marea rosa. Análise de Conjuntura opsa 8. Rio de Janeiro, Brasil: IUPERJ/UCAM.

PANizzA, F. (2009). Nuevas izquierdas y democracia en América Latina. Revista CIDOB d'Afers Inrernacionals, 85-86, 75-88.

Perrotta, D. (2013). La integración regional como objeto de estudio. De las teorías tradicionales a los enfoques actuales. En E. Llenderrozas (Ed.), Teoría de las Relaciones Internacionales (1-44). Buenos Aires, Argentina: EUDEBA. 
RiggirozZI, P. (2010). Region, regionness and regionalism in Latin America: towards a new synthesis. Red Latinoamericana de Política Comercial LATN, Working Paper 130. Buenos Aires, Argentina: FLACso.

Riggirozzi, P., y Tussie, D. (2012). The Rise of Post-hegemonic Regionalism. The case of Latin America. Londres, Reino Unido: Springer - United Nations University.

RiggirozZI, P., y Tussie, D. (2017). Rethinking our region in a post-hegemonic moment. En J. Briceño-Ruiz, e I. Morales (Eds.). Post-hegemonic regionalism in the Americas. Toward a Pacific-Atlantic Divide? (16-31). New York, EE. UU.: Routledge.

Sanahuja, J. A. 2008. Regionalismo e integración en América Latina: balance y perspectivas. Pensamiento Iberoamericano, 0, 75-106.

Sanahuja, J. A. 2010. La construcción de una región: Suramérica y el regionalismo posliberal. En M. Cienfuegos, y J. A. Sanahuja (Eds.). Una región en Construcción. Unasur y la integración de América del Sur . Barcelona, España: Funcadió CIDOB.

Sanahuja, J. A. 2012. Regionalismo post-liberal y multilateralismo en Sudámerica: el caso de Unasur. En A. Serbin, L. Martínez, y H. Ramanzini Júnior, Anuario de la Integración Regional de América Latina y el Gran Caribe 2012 (19-72). Buenos Aires, Argentina: CRIES.

Sanahuja, J. A. (2017). Beyond the Pacific-Atlantic divide. Latin American regionalism before a new cycle. En J. Briceño-Ruiz, e I. Morales (Eds.), Post-hegemonic regionalism in the Americas. Toward a Pacific-Atlantic Divide? (99-124). New York, EE. UU.: Routledge.

SARAIVA, M. G. (2011). Integração Regional na América do Sul: processos em aberto. En 3. ${ }^{\circ}$ Encontro Nacional da ABRI 2011. Governança Global e Novos Atores (p. 16). São Paulo, USP.

Sarti, I., Leite, M., Perrotta, D., y Cardoso, G. (Coords.) (2012). Por uma integração ampliada na América do Sul no século XXI. Rio de Janeiro, Brasil: Perse.

Silva, F. P. (2009). Vitórias na crise. Trajetórias das esquerdas latino-americanas contemporáneas (tesis de doctoral). IUPERJ, Rio de Janeiro, Brasil.

SiLva, F. P. (2010). Até onde vai a «onda rosa»? Análise de Conjuntura opsa 2. Rio de Janeiro, Brasil: IUPERJ/UCAM.

Solanas, F. (2011). Mercosur, Unasur y después... ¿Convergencia o competencia? Studia Politicae, 23, 11-29. 
182 La Integración Sudamericana en la encrucijada entre la ideología y el pragmatismo

SöDerbaum, F. (2016). Rethinking Regionalism. Londres, New York, Reino Unido, EE. UU.: Palgrave MacMillan UK.

Veiga, P. D., y Ríos, S. P. (2007). O regionalismo pós-liberal na América do Sul: origens, iniciativas e dilemas. Santiago de Chile, Chile: CEPAL.

El artículo ha sido desarrollado como parte de un proyecto de investigación financiado por CNPq y FAPERJ en el que Míriam Saraiva actúa como coordinadora. El presente artículo, en el que ambas autoras han tenido igual contribución, representa uno de sus resultados. 